\title{
Coherence and Persistent Currents in Mesoscopic Rings
}

\author{
Vinay Ambegaokar and Ulrich Eckern ${ }^{(a)}$ \\ Laboratory for Atomic and Solid State Physics, Clark Hall, Cornell University, Ithaca, New York 14853
}

(Received 1 March 1990)

\begin{abstract}
We calculate, to first order in the screened electron-electron interaction, the grand potential and the equilibrium current in a mesoscopic normal-metal ring threaded by a magnetic flux. The average current in an ensemble of such rings is found to be periodic in the flux with period $h / 2 e$, and to be given at zero temperature in order of magnitude by $I \sim e v_{F} l / L^{2}$, where $l$ is the mean free path and $L$ the circumference of the ring. Interference between time-reversed paths is the crucial ingredient. The effect decreases exponentially with temperature on a scale determined by the coherence energy $\sim h D / L^{2}$, where $D$ is the electronic diffusion constant. There is good agreement with recent measurements.

PACS numbers: $72.10 .-\mathrm{d}, 05.60 .+\mathrm{w}, 72.15 . \mathrm{Rn}$
\end{abstract}

In recent experiments, ${ }^{\prime}$ the magnetic response of a system of $10^{7}$ isolated, identically patterned copper rings has been shown to oscillate as a function of the enclosed magnetic flux on the scale of half a flux quantum. Here we calculate the contributions to this effect from electron coherence along time-reversed paths, familiar in the theory of weak localization, ${ }^{2.3}$ but here initiated by the electron-electron interaction. As regards both magnitude and temperature dependence, our calculation taken by itself is in satisfactory agreement with experiment. We think it likely that this is the primary explanation of the phenomenon.

Since our work differs qualitatively from recent attempts to predict and understand phenomena of this nature from a one-electron point of view, a few general remarks may be in order. The recent understanding of Aharonov-Bohm phenomena in various mesoscopic systems ${ }^{4.5}$ has made it clear that all physical properties of a single metal ring, of transverse dimensions much smaller than its circumference- if it is small enough, and cold enough, to permit single electrons to remain phase coherent around the ring-will be periodic in an applied magnetic flux with period $h / e$. In particular, fluxperiodic persistent currents of the order of $e v_{F} / L$, in the clean limit, were predicted some time ago. ${ }^{6}$ Recent work $^{7-9}$ has concentrated on analyzing the effect of impurity scattering on the one-electron effect, both in the (sample-specific) response of a single ring, and in the response of many rings measured simultaneously, as in Ref. 1, where a self-averaging reduction in the current per ring is to be expected. Even more recently, largescale numerical computations of the independentelectron contribution, which must be added to our result, have been performed. ${ }^{10} \mathrm{~A}$ quantitative comparison of these effects follows a description of our calculation.

To put our calculation in perspective, it is useful to recall the first work on the periodicity of the magnetoconductivity of cylinders, which predicted ${ }^{11}$ and observed ${ }^{4}$ a period of $h / 2 e$, henceforth called $\Phi_{0}$. Here the third dimension is long enough so that one is effectively taking an average over many rings, thereby washing out the $h / e$ effect and only leaving the interference between a given circumnavigation and its time-reversed counterpart. ${ }^{11,12}$ Our calculation is greatly influenced by this understanding, but there is one important difference. Whereas for the conductivity it is possible to imagine a low-energy electron-hole pair being created by the electric field, the equilibrium effect we are calculating here seems to require a fluctuation produced by the electron-electron interactions. ${ }^{13}$ (We have looked for, and failed to find, a backscattering interference effect when this interaction is neglected.)

Consider, then, a ring of circumference $L$ and transverse dimensions $L_{\perp} \ll L$ in a magnetic field. Keeping in mind the experimental situation, $L \sim 1 \mu \mathrm{m}, l=v_{F} \tau$ $\sim L_{\perp} \sim 100 \AA$, we suppose that the following inequalities are satisfied:

$$
\hbar D / L^{2} \ll \hbar v_{F} / L \ll \hbar / \tau \ll \epsilon_{F},
$$

where $D$ is the diffusion constant $v_{F}^{2} \tau / 3, v_{F}$ and $\epsilon_{F}$ are the Fermi velocity and energy, respectively, and $\tau$ is the relaxation time for elastic scattering. The temperature regime of interest is $T \lesssim \hbar D / L^{2} \sim 100 \mathrm{mK}$. The condition $\hbar v_{F} / L \ll \hbar / \tau$ allows one to neglect the discreteness of the single-particle levels. The last inequality in Eq. (1), which says that the de Broglie wavelength is much smaller than all other length scales, means that we are dealing with a mesoscopic and not a microscopic system.

In the following, we consider the backscattering corrections to the impurity-averaged thermodynamic potential of the system. We work in the grand-canonical ensemble, but have carefully examined, and found negligible, the dependence of the mean number of electrons on the applied flux. To first order in the screened electronelectron interaction, the grand potential $\Omega$ is given by

$$
\Omega=\Omega_{0}+\langle\boldsymbol{V}\rangle_{0},
$$

where the subscript 0 refers to the system with no interactions between electrons but fully including the impurity potential, and the brackets mean a thermodynam- 
ic average, which produces direct (Hartree) and exchange (Fock) contributions. We average over the distribution of impurities in the usual way, and only keep terms corresponding to multiple scattering of the pair excitation, as indicated in Fig. 1. The plan is to evaluate the contribution of these graphs, in the presence of a magnetic flux $\Phi$ and calculate the current from the equation

$$
I=-\partial \Omega / \partial \Phi,
$$

valid for an isolated ring. We use the standard Matsubara method. Both graphs have two independent Matsubara variables which we take to be $\zeta_{l} \equiv(2 l+1) \pi T i$ and $\zeta_{l}-v_{m}$, with $v_{m} \equiv 2 m \pi T i$, and $l, m=0, \pm 1, \pm 2, \ldots T$ is in units of energy. In the following, we omit the subscripts to simplify writing.

First, consider the case of no magnetic field. Then the electron states can be labeled by momentum and spin, as illustrated in Fig. 1. The particle-particle ladder, denoted by $K$, is readily evaluated. In the long-wavelength, low-frequency limit, and in the region $\operatorname{Im}(\zeta) \operatorname{Im}(\zeta-v)$ $<0$, it has the well-known diffusive form:

$$
K(q, v, \zeta)=\frac{1}{2 \pi N(0) \tau^{2}} \frac{1}{|v|+D q^{2}} .
$$

The Hartree and Fock contributions to the grand potential are

$$
\mathcal{V}^{-1} \Omega_{H}=2 T^{2} \sum_{\zeta, .} \int d \mathbf{p} d \mathbf{p}^{\prime} d \mathbf{q} V\left(\mathbf{p}-\mathbf{p}^{\prime}, 0\right) K(\mathbf{q}, v, \zeta) \times G(\mathbf{p}, \zeta) G(\mathbf{p}-\mathbf{q}, \zeta-v) \times G\left(\mathbf{p}^{\prime}, \zeta\right) G\left(\mathbf{p}^{\prime}-\mathbf{q}, \zeta-v\right),
$$

and

$$
\mathcal{V}^{-1} \Omega_{F}=-T^{2} \sum_{\zeta, v} \int d \mathbf{p} d \mathbf{p}^{\prime} d \mathbf{q} V\left(\mathbf{p}+\mathbf{p}^{\prime}-\mathbf{q}, v\right) K(\mathbf{q}, v, \zeta) \times G(\mathbf{p}, \zeta) G(\mathbf{p}-\mathbf{q}, \zeta-v) \times G\left(\mathbf{p}^{\prime}, \zeta-v\right) G\left(\mathbf{p}^{\prime}-\mathbf{q}, \zeta\right),
$$

where $\mathcal{V}$ is the volume of the system.

Since small $\mathbf{q}$ are emphasized by $K(\mathbf{q})$, we may neglect the $\mathbf{q}$ dependence elsewhere in Eqs. (5) and (6). We now integrate over $\mathbf{p}$ and $\mathbf{p}^{\prime}$, using

$$
\int d \mathbf{p} \simeq N(0) \int d \xi \frac{d \Omega}{4 \pi} .
$$

Note that the $\xi$ and $\xi^{\prime}$ integrations are only nonzero when the inequality written above Eq. (4) is obeyed. One thus obtains

$$
\Omega_{H}+\Omega_{F}=2 \pi N(0) T^{2} \sum_{v} \sum_{\zeta}^{\prime}\left\langle 2 V\left(\mathbf{p}-\mathbf{p}^{\prime}, 0\right)-V\left(\mathbf{p}+\mathbf{p}^{\prime}, v\right)\right\rangle\left[\frac{1}{|v| \tau+1}\right]^{2} \sum_{\mathbf{q}} \frac{1}{|v|+D q^{2}} .
$$

Here, the sum over $\zeta$ is restricted as just discussed, and the brackets mean an angular average, with $\mathbf{p}$ and $\mathbf{p}^{\prime}$ at the Fermi surface. We take $V$ to be the screened Coulomb interaction, which in the regime $v, D q^{2} \ll 1 / \tau$ is given by

$$
V(\mathbf{q}, v)=\frac{4 \pi e^{2}}{q^{2}} \frac{|v| / D+q^{2}}{q_{\mathrm{TF}}^{2}+q^{2}+|v| / D},
$$

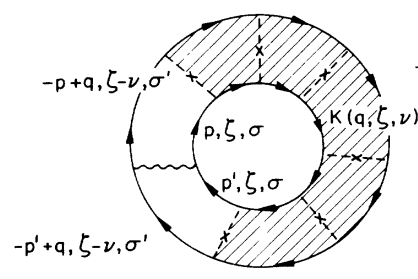

(a)

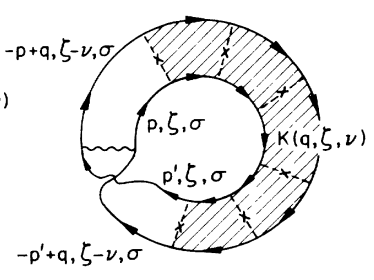

(b)
FIG. 1. Feynman diagrams corresponding to (a) the Hartree and (b) the Fock contributions to the grand potential. The wavy line refers to the screened Coulomb interaction, and the dashed lines with crosses to the impurity scattering averaged over impurity positions. with $q_{\mathrm{TF}}$ the Thomas-Fermi wave number given by $q \underset{\mathrm{TF}}{2}=8 \pi e^{2} N(0)$. Equation (9) is not correct for $q \approx k_{F}$, but if we estimate the average using it we obtain a result that is (almost) independent of $|v|$, even when this variable is of the order of $\tau^{-1}$. Thus we replace each of the average potentials in Eq. (8) by $\bar{V}=\left\langle V\left(\mathrm{p}-\mathrm{p}^{\prime}, 0\right)\right\rangle$. The restricted sum over $\zeta$ in Eq. (8) leads to a factor $|v| / 2 \pi T$, so that we obtain the simple expression

$$
\Delta \Omega \equiv \Omega_{H}+\Omega_{F}=N(0) \bar{V} T \sum_{\mathbf{q} . v} \frac{|v|}{|v|+D q^{2}} \frac{1}{(1+|v| \tau)^{2}} .
$$

The Matsubara sum can be done using standard methods and yields

$$
\Delta \Omega=-N(0) \bar{V} \frac{1}{\pi} \int_{0}^{\infty} d E \operatorname{coth} \frac{E}{2 T} \sum_{\mathbf{q}} \frac{\hbar D q^{2} E}{E^{2}+\left(\hbar D q^{2}\right)^{2}} .
$$

Here, we have neglected the last factor in Eq. (10), so that an upper cutoff of the order of $\hbar / \tau$ is implicit.

Now, as in the theory of the oscillatory magnetoconductivity, an applied magnetic field is accounted for by 
replacing $D q^{2}$ with the eigenvalues of the operator

$$
D\left(\frac{1}{i} \nabla-\frac{2 e}{\hbar} A\right)^{2}
$$

subject to the effective boundary condition of zero slope at the surfaces. ${ }^{14}$ For the conditions given in Eq. (1), the transverse dimensions are then frozen out, and the rule for summing over $\mathbf{q}$ is

$$
\sum_{\mathbf{q}} f(\mathbf{q}) \rightarrow \sum_{n=-\infty}^{\infty} f\left(\frac{2 \pi}{L}\left(n-\frac{\Phi}{\Phi_{0}}\right)\right),
$$

which is a periodic function of the flux $\Phi$ with period $\Phi_{0}=h / 2 e$. Applying this rule to Eq. (11), and introducing the variables $\Theta=T L^{2} / D \hbar(2 \pi)^{2}$, and $x=\Phi / \Phi_{0}$, we arrive at

$$
\Delta \Omega=\frac{N(0) \bar{V}}{\pi}\left(\frac{2 \pi}{L}\right)^{2} \hbar D g(x)
$$

with

$g(x)=-\int_{0}^{\infty} d y \operatorname{coth}\left(\frac{y}{2 \Theta}\right) \sum_{n=-\infty}^{\infty} \frac{y(n-x)^{2}}{y^{2}+(n-x)^{4}}$.

Now, $g(x)$, being even and periodic in $x$ with period unity, has the expansion

$$
g(x)=g_{0}+2 \sum_{m=1}^{\infty} g_{m} \cos (2 \pi m x) .
$$

Inverting this Fourier series, one obtains

$$
\begin{aligned}
g_{m} & =-\int_{0}^{\infty} d y y \operatorname{coth} \frac{y}{2 \Theta} \int_{-\infty}^{\infty} d x \frac{x^{2}}{y^{2}+x^{4}} e^{2 \pi I m x} \\
& =\frac{1}{2 \pi^{2} m^{3}} \int_{0}^{\infty} d z z^{2} \operatorname{coth}\left(\frac{z^{2}}{\Theta_{m}}\right) e^{-z}[\sin z-\cos z],
\end{aligned}
$$

where $\Theta_{m}=T / T_{m}, T_{m} \equiv\left(\hbar D / L^{2}\right) m^{-2}$. In the limit of zero temperature, $g_{m}(T=0)=\left(2 \pi^{2} m^{3}\right)^{-1}$. Note that $g_{m}$ decreases rapidly when $T>T_{m}$, and that the higher harmonics die out more quickly because $T_{m} \propto m^{-2}$. A numerical evaluation of the integral in Eq. (16) yields the curve for $g_{m}(T) / g_{m}(0)$ shown in Fig. 2. In the range shown, this curve is quite well approximated by the function $\exp \left(-T / 3 T_{m}\right)$. From $T / T_{m} \sim 10$, the asymptotic - but presumably somewhat academic from an experimental point of view-behavior obtained from (16) takes over and is given by

$$
\left[\frac{1}{2}\left(\pi T / T_{m}\right)^{3}\right]^{1 / 2} \exp \left[-\sqrt{2 \pi T / T_{m}}\right] .
$$

From Eqs. (3) and (13)-(15), the average current in a single ring is

$$
I=\frac{N(0) \bar{V}}{\pi}\left(\frac{2 \pi}{L}\right)^{2} \hbar D \frac{4 \pi}{\Phi_{0}} \sum_{m=1}^{\infty} m g_{m} \sin \left(2 \pi \frac{\Phi}{\Phi_{0}} m\right) .
$$

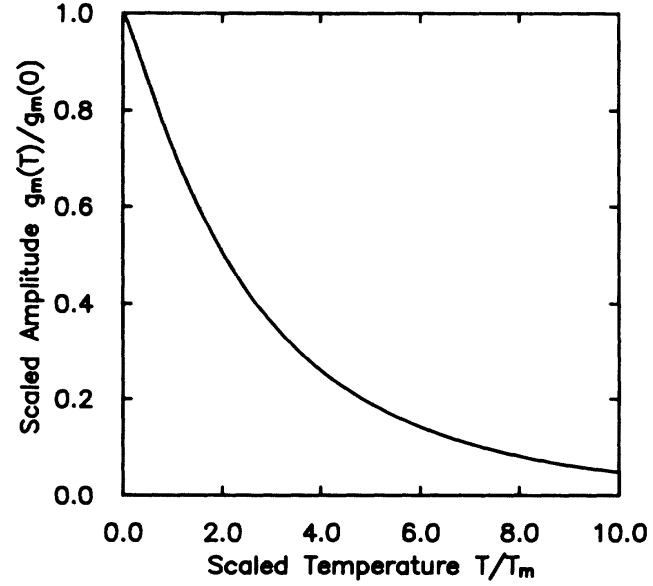

FIG. 2. The temperature dependence of the amplitude of the equilibrium current in scaled form, as explained in the text.

In particular, for the first harmonic Eq. (17) reduces to

$$
\begin{aligned}
I & =\frac{8}{3 \pi} N(0) \bar{V} e \frac{v^{\prime} l}{L^{2}} \frac{g_{1}(T)}{g_{1}(0)} \sin \left(2 \pi \frac{\Phi}{\Phi_{0}}\right) \\
& =\left[\frac{8 N(0) \overline{V l}}{3 \pi L}\right] \frac{e v_{F}}{L} \exp \left(-\frac{T}{3 T_{1}}\right) \sin \left(2 \pi \frac{\Phi}{\Phi_{0}}\right),
\end{aligned}
$$

where in the last line we have made the approximation suggested in the last paragraph, and extracted a dimensionless constant from the prefactor.

Let us now compare this theory with the experiment. ' The observed maximum current corresponding to period $\Phi_{0}$ is quoted in the form $3 \times 10^{-3}\left(e v_{F} / L\right) \exp [-T /(80$ $\mathrm{mK})$ ].

The estimate suggested in and below Eq. (9) yields

$$
2 N(0) \bar{V}=\frac{\left\langle V\left(\mathrm{p}-\mathrm{p}^{\prime}, 0\right)\right\rangle}{V(0,0)}=\frac{\alpha}{2} \ln \left(\frac{2+\alpha}{\alpha}\right),
$$

where $2 \alpha=\left(q_{\mathrm{TF}} / k_{F}\right)^{2}$. Using standard parameters for copper, including the effective-mass enhancement, ${ }^{15}$ we estimate $\alpha \sim 1.2$, and thus $2 N(0) \bar{V} \sim 0.6$. In Ref. 1 , one finds $l=200 \AA$, and $L=2.2 \mu \mathrm{m}$, and one can deduce from that paper that the accepted estimate $v_{F}=1.6 \times 10^{8}$ $\mathrm{cm} / \mathrm{sec}$ for the Fermi velocity has been used. With these numbers we find that the dimensionless factor in square brackets in the last line of Eq. (18) is $\simeq 2.2 \times 10^{-3}$, and that the characteristic temperature $3 T_{1}=3 D \hbar / L^{2}$ is 50 mK. ${ }^{16}$

Finally, we must address a concern that this calculation may raise. Does it make sense to calculate so small a current using the grand-canonical ensemble? This question is made particularly cogent because of recent calculations ${ }^{9}$ which neglect the electron-electron interaction but suggest that while the first harmonic (period $2 \Phi_{0}$ in our notation) is strongly suppressed on impurity averaging, the second harmonic (period $\Phi_{0}$ ) is finite, but only when each member of the ensemble is kept at fixed 
particle number, whereas a calculation at fixed chemical potential leads to a washing out of all harmonics. In the regime of interest here, $l \ll L \ll M l$, where $M=\left(k_{F} L_{\perp}\right)^{2}$ is the number of transverse channels, these authors obtain $^{10}$ a zero-temperature current proportional to $\sqrt{l / M L}$ - different from our $M$-independent result - which they estimate to be a factor of 20 smaller than the experiment. 'The temperature dependence is not addressed in these numerical calculations.

As regards our calculation, we have checked that there is no sensitivity of the average number of electrons to the flux at fixed chemical potential. This average is obtained by taking the derivative of the grand potential, given by Fig. 1, with respect to the chemical potential. This procedure successively replaces each Green's function by its square. Upon $\xi$ integration, we then find an expression that is odd in $\zeta$ and vanishes on summation. This result may seem surprising, since it is known that the density of states at the Fermi surface is modified by these contributions. However, there is an exact compensation from regions far from the Fermi surface.

In conclusion, we have identified a contribution to the average persistent current in an ensemble of rings, which depends on the existence of interactions between electrons, exploits interference effects known from the theory of weak localization, and, taken by itself, is in good agreement with experiment. Our zero-temperature current is estimated to be somewhat smaller than the experiment, so that we cannot rule out the possibility that single electron effects also give some contribution. Finally, it is worth noting, for future experiments, that the sign of (17) may change for a dirty superconductor above its transition temperature.

This work is supported in part by NSF Grant No. DMR-88 15828. U.E. acknowledges a Heisenberg Fellowship through the Deutsche Forschungsgemeinschaft. We thank B. L. Altshuler, D. P. DiVincenzo, G. Montambaux, and E. K. Riedel for suggestions and useful information.

\footnotetext{
(a) Present address: Kernforschungszentrum Karlsruhe, Institut für Nukleare Festkörperphysik, Postfach 3640, 7500
}

Karlsruhe, Federal Republic of Germany.

'L. P. Levy, G. Dolan, J. Dunsmuir, and H. Bouchiat, Phys. Rev. Lett. 64, 2074 (1990).

2G. Bergmann, Phys. Rep. 107, 1 (1984).

${ }^{3}$ P. A. Lee and T. V. Ramakrishnan, Rev. Mod. Phys. 57, 287 (1985).

${ }^{4}$ D. Yu. Sharvin and Yu. V. Sharvin, Pis'ma Zh. Eksp. Teor. Fiz. 34, 285 (1981) [JETP Lett. 34, 272 (1981)].

${ }^{5}$ R. A. Webb et al., Phys. Rev. Lett. 54, 2696 (1985); S. Datta et al., Phys. Rev. Lett. 55, 2344 (1985); G. Timp et al., Phys. Rev. Lett. 58, 2814 (1987).

${ }^{6} \mathrm{M}$. Büttiker, Y. Imry, and R. Landauer, Phys. Lett. 96A, 365 (1983).

${ }^{7}$ H. F. Cheung, E. Riedel, and Y. Gefen, Phys. Rev. Lett. 62, 587 (1989).

${ }^{8}$ N. Trivedi and D. A. Browne, Phys. Rev. B 38, 9581 (1988).

${ }^{9}$ H. Bouchiat and G. Montambaux, J. Phys. (Paris) 50, 2695 (1989).

${ }^{10} \mathrm{G}$. Montambaux et al. (to be published).

${ }^{\prime \prime}$ B. L. Altshuler, A. G. Aronov, and B. Z. Spivak, Pis'ma Zh. Eksp. Teor. Fiz. 33, 101 (1981) [JETP Lett. 33, 94 (1981)].

${ }^{12}$ For a review that emphasizes this physics, see S. Chakravarty and A. Schmid, Phys. Rep. 140, 193 (1986).

${ }^{13}$ B. L. Altshuler, D. E. Kmelnitskii, and B. Z. Spivak, Solid State Commun. 48, 841 (1983). In this paper, weaklocalization corrections to the supercurrent in superconductor-normal-metal-superconductor junctions are discussed. For a detailed review, see also B. L. Altshuler and A. G. Aronov, in Electron-Electron Interactions in Disordered Systems, edited by A. L. Efros and M. Pollak (North-Holland, Amsterdam, 1985).

${ }^{14} \mathrm{~A}$ readable discussion of how effective boundary conditions for moment expansions are obtained from the underlying integral equations, albeit in another context, is to be found in Chap. 7 of P. G. de Gennes, Superconductivity of Metals and Alloys (Benjamin, New York, 1966).

${ }^{15}$ N. W. Ashcroft and N. D. Mermin, Solid State Physics (Holt, Reinhart and Winston, New York, 1976), pp. 5, 48, and 342.

${ }^{16}$ The multiple-scattering corrections considered in Refs. 13 would, for a repulsive potential such as (19), reduce the effective interaction in the Cooper channel. We find this effect difficult to estimate; a naive calculation gives a reduction of the order of a factor of 3 , thereby worsening the agreement for the magnitude of the current. 